\title{
CONSIDERACIONES PARA LA APLICACIÓN DEL MÉTODO HIDRÁULICO R2CROSS PARA ESTIMAR CAUDALES ECOLÓGICOS EN COLOMBIA
}

\author{
CONSIDERATIONS REGARDING THE APPLICATION OF R2CROSS HYDRAULIC \\ METHOD TO ESTIMATE ENVIRONMENTAL FLOWS IN COLOMBIA
}

David Gallo-Vélez

\begin{abstract}
RESUMEN
Colombia es un país privilegiado en cuanto a la oferta hídrica, sin embargo, la mayor parte de ésta se encuentra en zonas poco pobladas y la fracción de agua superficial, disponible para las áreas de mayor concentración poblacional, se ve afectada por la sobreexplotación y la contaminación de los ríos. Por esta razón, se sugiere la estimación del caudal ecológico como un instrumento para el manejo de las cuencas del país. Dentro de este trabajo de revisión bibliográfica se presentan los principales conceptos asociados al caudal ecológico, las diferentes metodologías utilizadas para su estimación y se analizan los antecedentes en Colombia. Posteriormente, se hace énfasis en el método hidráulico R2CROSS, se mencionan sus bases teóricas y se muestra el caso de estudio de los ríos Assabet y Charles en Massachusetts (Estado Unidos). A partir de allí, se hizo un comparativo con las condiciones locales para definir las ventajas y desventajas de la aplicación de este método en Colombia. Algunas de las ventajas identificadas fueron la relación costo-beneficio, el poco tiempo requerido para su aplicación, la obtención de resultados similares a los de métodos más complejos como el de la Instream Flow Incremental Methodology (IFIM) y la conveniencia para usarlo en lugares con información hidrológica incompleta. Por otro lado, dentro de sus desventajas se encuentra un mayor costo que los métodos hidrológicos, los sesgos derivados de suponer que a partir de los parámetros hidráulicos se pueden determinar las condiciones óptimas para todas las especies del ecosistema acuático y rivereño y, finalmente, su precisión que depende de una correcta elección de la sección del río.
\end{abstract}

PALABRAS CLAVE: Caudal ecológico, método hidráulico, R2CROSS, HEC-RAS, Colombia.

\begin{abstract}
Despite Colombia is a privileged country in terms of water supply, most of such supply is located in sparsely-populated areas; the surface water fraction, available for higher populated areas, is affected by both overexploitation and pollution of rivers. Therefore, an estimation of environmental flow is suggested, as a means of managing the country's river basins. First, this literature review paper presents the main concepts associated with environmental flow and the several methods used for its estimation, as well as a background analysis in Colombia. Subsequently, it highlights the R2CROSS hydraulic method, including its theoretical bases, and it shows the study case of Assabet and Charles rivers in Massachusetts (United States). From that point, a comparative against local conditions was performed, in order to define the advantages and disadvantages of applying said method in Colombia. Some of the advantages found were the cost-benefit ratio, the little time required for its application, the obtaining of similar results to those gotten by more complex methods such as Instream Flow Incremental Methodology (IFIM), and the convenience to use it in places with incomplete hydrological information. On the other hand, some of the disadvantages found were a higher cost than hydrological methods, the biases derived from assuming that the hydraulic parameters can determine the optimal conditions for every species of aquatic and riverine ecosystem, and the precision of such method, which depends on a correct choice of the river section.
\end{abstract}

KEY WORDS: Environmental Flow, Hydraulic Method, R2CROSS, HEC-RAS, Colombia. 


\section{INTRODUCCIÓN}

El constante crecimiento de la población mundial y el consecuente aumento progresivo de la demanda hídrica, han generado la sobreexplotación del recurso, a tal punto que muchos ríos se han secado o presentan condiciones precarias por los altos niveles de contaminación que impiden su uso como una fuente de abastecimiento. Así, por ejemplo, según datos del Estudio Nacional del Agua 2014, las proyecciones para Colombia en el 2022, estiman una demanda hídrica total de 63717 millones de $\mathrm{m}^{3}$ lo que equivale a un incremento del 77,1 \% con respecto a la demanda durante el 2012 que fue de 35987,1 millones de $\mathrm{m}^{3}$ (IDEAM, 2015).

Colombia es un país privilegiado en cuanto a los recursos hídricos disponibles. Sin embargo, gran parte de esta oferta no se encuentra en las regiones donde se han desarrollado las principales actividades socioeconómicas y donde se ha dado una mayor concentración poblacional. Además de esto, las fuentes de agua están sometidas a sobreexplotación o reciben altas cargas contaminantes, lo que deteriora significativamente la calidad del agua e impide su uso por parte de la población. Es decir, a pesar de la oferta disponible, la fracción aprovechable resulta cada vez menor (Ministerio de Ambiente Vivienda y Desarrollo Territorial, 2010).

A pesar de lo anterior, solo hasta el año 2010 se estableció en Colombia una Política Nacional para la Gestión Integral del Recurso Hídrico, basada en un diagnóstico de la oferta, demanda y problemáticas asociadas al agua en nuestro país. Así mismo, el Ministerio de Ambiente, Vivienda y Desarrollo Territorial (MAVDS) estableció objetivos, metas y líneas de acción estratégicas, para lograr una gestión integral del recurso, dentro de las cuales se encuentra la definición de los "caudales mínimos necesarios para el mantenimiento de las corrientes superficiales y sus ecosistemas acuáticos asociados, e implementar medidas para garantizarlos" (Ministerio de Ambiente Vivienda y Desarrollo Territorial, 2010).

Los caudales ecológicos constituyen un instrumento para la gestión integral del recurso hídrico dado que a partir de la aplicación de metodologías con bases científicas y técnicas sólidas, se logra estimar, de manera objetiva, el caudal mínimo necesario para la conservación de los ecosistemas asociados a un río. De igual manera se pueden definir planes de manejo para la cuenca evaluada, de tal forma que el uso se haga de manera sostenible, permitiendo el desarrollo de los individuos sin generar impactos significativos en el medio biofísico.

La elección de un método para estimar caudales ecológicos depende de aspectos como la inmediatez del problema, los recursos disponibles (tanto técnicos como económicos), la importancia del rio, la complejidad del sistema, entre otros (O'Keeffe y Quesne, 2010). Además se deben considerar las condiciones específicas de la región donde se realice la valoración. Por estas razones, es claro que no se puede definir un método universal para la estimación de caudales ambientales.

Los métodos hidráulicos constituyen una alternativa eficiente en cuanto al costo y facilidad de aplicación, puesto que arrojan resultados similares a los de métodos ecohidráulicos más complejos como la metodología IFIM (Instream Flow Incremental Methodology, Armstrong et al., 2001).

En este artículo se hará una introducción al método hidráulico R2CROSS, como alternativa para la estimación de caudales ambientales en Colombia, se evaluarán las ventajas y desventajas que podrían presentarse al aplicarlo en un país de clima tropical, muy diferente al que se tiene en el lugar que fue desarrollado del método (Panel de Conservación de Agua de Colorado - Estados Unidos en el año de 1996).

\section{Definición de caudal ecológico}

Para el año 2004, se definió el caudal ecológico como aquel que permitiera el mantenimiento del patrimonio hidrobiológico y sociocultural del medio fluvial y que, a su vez, fuera compatible con la necesidad de abastecimiento doméstico y de suministro agrícola e industrial (Magdaleno-Mas, 2004). Una definición más reciente, habla de caudal ecológico como el flujo mínimo necesario para preservar los valores ecológicos del cauce, dentro de los cuales se incluyen los hábitats naturales, la función de dilución de contaminantes, el uso recreativo y el paisaje, entre otros (Endesa Chile, 2011).

Para una mejor comprensión del concepto de caudal ecológico, en la Tabla 1 se presenta un resumen de las diferentes definiciones encontradas en la literatura. Sin embargo, tal como lo plantea Consuegra-Martínez (2013), la mayoría de ellas coinciden en aspectos como:

- Cantidad: "Caudal mínimo requerido para el normal funcionamiento de un ecosistema" 
- Calidad: "Caudal que garantiza una calidad específica del recurso hídrico para el normal desarrollo del ecosistema acuático, así como para su uso en actividades de índole socioeconómica”

- Variabilidad temporal y espacial: Es un caudal que varía en función de las condiciones del cauce.

- Instrumento de planeación: utilizado para el ordenamiento del recurso hídrico.

\section{Enfoques metodológicos para la estimación de caudales ecológicos}

En la actualidad existen más de 200 métodos para estimar caudales ecológicos (Díez-Hernández, 2005). Varios autores entre los que podemos mencionar a Magdaleno-Mas (2004); Agualimpia-Dualiby y CastroMéndez (2006); Castro-Heredia et al. (2006); Endesa Chile (2011); Izquierdo-Santacruz y Madroñero-Palacios (2014) y Ocampo et al. (2014) coinciden en que las principales metodologías se agrupan en cuatro (04) enfoques: hidrológicos, de simulación de hábitat, holísticos e hidráulicos. A continuación, en la Tabla 2 se presenta un resumen de los principales métodos que se incluyen dentro de los cuatro enfoques metodológicos mencionados.

En los últimos años, también han tomado fuerza los métodos que involucran la calidad del agua para determinar el caudal ecológico, sin embargo, aún no han alcanzado el estatus y la difusión de las 4 metodologías mencionadas con anterioridad (Rodríguez et al., 2008; Madroñero-Palacios y Mafla-Chamorro, 2013). Por ejemplo, China es un país donde el recurso hídrico se ve afectado seriamente por la contaminación, en consecuencia, los caudales ecológicos no pueden ser determinados solo con un enfoque de cantidad, también es necesario considerar la calidad del agua y los regímenes naturales de caudal como factores determinantes para establecer caudales que logren el propósito de conservar los ecosistemas acuáticos (Liu et al., 2016).

\section{Antecedentes de la estimación de caudales ecológicos en Colombia}

En Colombia, la temática del caudal ecológico y todo lo que se relaciona con ésta, al igual que importante, es novedosa. A diferencia de otros países del mundo, donde existen políticas que incluyen el concepto de caudal ecológico desde la década de los ochenta, en nuestro país se empezó a abordar a partir del año 2000 (Consuegra-Martínez, 2013). Pese a esto, Colombia es el país sudamericano que más ha avanzado en el desarrollo del tema y es el único que cuenta con una propuesta metodológica propia para la estimación de caudales ecológicos (Rodríguez-Gallego et al., 2011).

En el año 2005, con el proyecto de Ley del Agua (P.L. 365 de 2005 C), se tuvo el primer intento por sentar las bases respecto al tema del caudal ecológico en Colombia. El artículo 21 de este proyecto designaba al IDEAM como encargado de diseñar los lineamientos en materia de caudal ecológico. Mientras esto ocurría, las estimaciones de caudal ecológico debían realizarse con métodos estrictamente hidrológicos. El primero de ellos tiene en cuenta el caudal medio diario de 5 a 10 años, cuya duración es mayor del $97,5 \%$, que se comprueba y corresponde a un periodo de retorno de 2,3 años. El segundo método define el caudal mínimo como un porcentaje en torno al $25 \%$ del caudal medio mensual multianual menor del cauce estudiado. Sin embargo, lo anterior no dejó de ser un proyecto y nunca fue aprobado por el Congreso de la Republica (IzquierdoSantacruz y Madroñero-Palacios, 2014; Ministerio de Ambiente Vivienda y Desarrollo Territorial, 2010).

Solo hasta el año 2007, se hace evidente un interés del estado colombiano por definir una metodología para el cálculo del caudal ecológico. Para esto, se suscribe un convenio interadministrativo entre el antiguo Ministerio de Ambiente, Vivienda y Desarrollo Territorial (MAVDT) y la Universidad Nacional de Colombia. Este convenio, tenía como objetivo la elaboración de una propuesta metodológica para la estimación de caudales ambientales que fuera adaptada al contexto local y que tuviera en cuenta y corrigiera las limitaciones de otros métodos aplicados alrededor del mundo. Producto de este convenio, en el 2008 se publica el informe final denominado "Metodología para la estimación del caudal ambiental en proyectos licenciados" con todos los detalles de la metodología desarrollada (Rodríguez et al., 2008), a partir del cual se construye la versión de la Autoridad Nacional de Licencias Ambientales (ANLA): "Metodología para la estimación y evaluación del caudal ambiental en proyectos que requieren licencia ambiental”, publicada en 2013.

Todavía para el año 2013, Colombia no contaba con ningún marco legal que definiera las pautas para el cálculo de los caudales ecológicos. Simplemente contaba con la metodología propuesta por la ANLA que no tenía un carácter normativo y estaba sujeta a las modificaciones que se consideraran pertinentes después de evaluarla en el contexto local (Autoridad Nacional de Licencias Ambientales, 2013). 
Tabla 1. Conceptos y definiciones relacionadas con el caudal ambiental.

\begin{tabular}{|c|c|c|}
\hline Denominación & Concepto & Referencia \\
\hline Caudal Ambiental & $\begin{array}{l}\text { Es el régimen hídrico que se establece en un río, humedal o zona costera } \\
\text { para sustentar ecosistemas y sus beneficios donde hay usos del agua } \\
\text { que compiten entre sí y donde los caudales están regulados. El caudal } \\
\text { ambiental es usado para valorar cuánta agua puede quitársele al río sin } \\
\text { causar un nivel inaceptable de degradación del ecosistema ribereño en } \\
\text { el caso de ríos gravemente alterados. Se considera caudal ambiental } \\
\text { la cantidad de agua necesaria para restablecer el río y rehabilitar el } \\
\text { ecosistema hasta un estado o condición requerida. }\end{array}$ & $\begin{array}{l}\text { King y Louw (1998); Palau } \\
\text { (1994); Dyson et al. (2003) }\end{array}$ \\
\hline $\begin{array}{l}\text { Régimen de Caudal } \\
\text { Ambiental }\end{array}$ & $\begin{array}{l}\text { Es aquel que permite cumplir con una condición establecida del } \\
\text { ecosistema ribereño. En él se detallan caudales específicos en magnitud, } \\
\text { periodicidad, frecuencia y duración, tanto de caudales basales como de } \\
\text { avenidas y crecientes en la escala de variabilidad intra e interanual, todo } \\
\text { ello diseñado para mantener en funcionamiento todos los componentes } \\
\text { del ecosistema. }\end{array}$ & King et al. (1999) \\
\hline Caudal Ecológico Mínimo & $\begin{array}{l}\text { Caudal que restringe el uso durante las estaciones de caudales bajos y } \\
\text { mantienen la vida en el río. No aporta una solución ecológica. Se calcula } \\
\text { de forma directa y arbitraria, producto de un pacto más que de una } \\
\text { formulación científica. }\end{array}$ & $\begin{array}{l}\text { King et al. (1999); Palau } \\
\text { (2003) }\end{array}$ \\
\hline Caudal de Mantenimiento & $\begin{array}{l}\text { Caudal requerido para mantener todas las funciones ecosistémicas } \\
\text { del río, incluyendo la incorporación continua y balanceada de las } \\
\text { especies acuáticas y riparias. Es un caudal calculado y dirigido hacia la } \\
\text { conservación de los valores bióticos del ecosistema fluvial. }\end{array}$ & APROMA (2000) \\
\hline $\begin{array}{l}\text { Caudal de } \\
\text { Acondicionamiento }\end{array}$ & $\begin{array}{l}\text { Se refiere a un caudal que puede establecerse como complemento de } \\
\text { caudales mínimos o de mantenimiento, para una finalidad concreta, } \\
\text { ajena a la conservación de valores bióticos del ecosistema fluvial y } \\
\text { referida a aspectos abióticos (dilución, paisaje, usos recreativos, etc.). }\end{array}$ & Palau (2003) \\
\hline Caudal Ecológico & $\begin{array}{l}\text { Caudal mínimo necesario en una fuente o curso fluvial, para preservar } \\
\text { la conservación de los ecosistemas fluviales actuales, en atención a los } \\
\text { usos de agua comprometidos, a los requerimientos físicos de la corriente } \\
\text { fluvial, para mantener su estabilidad y cumplir sus funciones tales como, } \\
\text { dilución de contaminantes, conducción de sólidos, recarga de acuíferos y } \\
\text { mantenimientos de las características paisajísticas del medio. }\end{array}$ & Ormazabal (2004) \\
\hline Caudal de Compensación & $\begin{array}{l}\text { Caudal mínimo necesario para asegurar la supervivencia de un } \\
\text { ecosistema acuático preestablecido. }\end{array}$ & UNESCO (s.f.) \\
\hline
\end{tabular}

Fuente: Rodríguez et al. (2008). 
Tabla 2. Enfoques metodológicos para la estimación de caudales ecológicos.

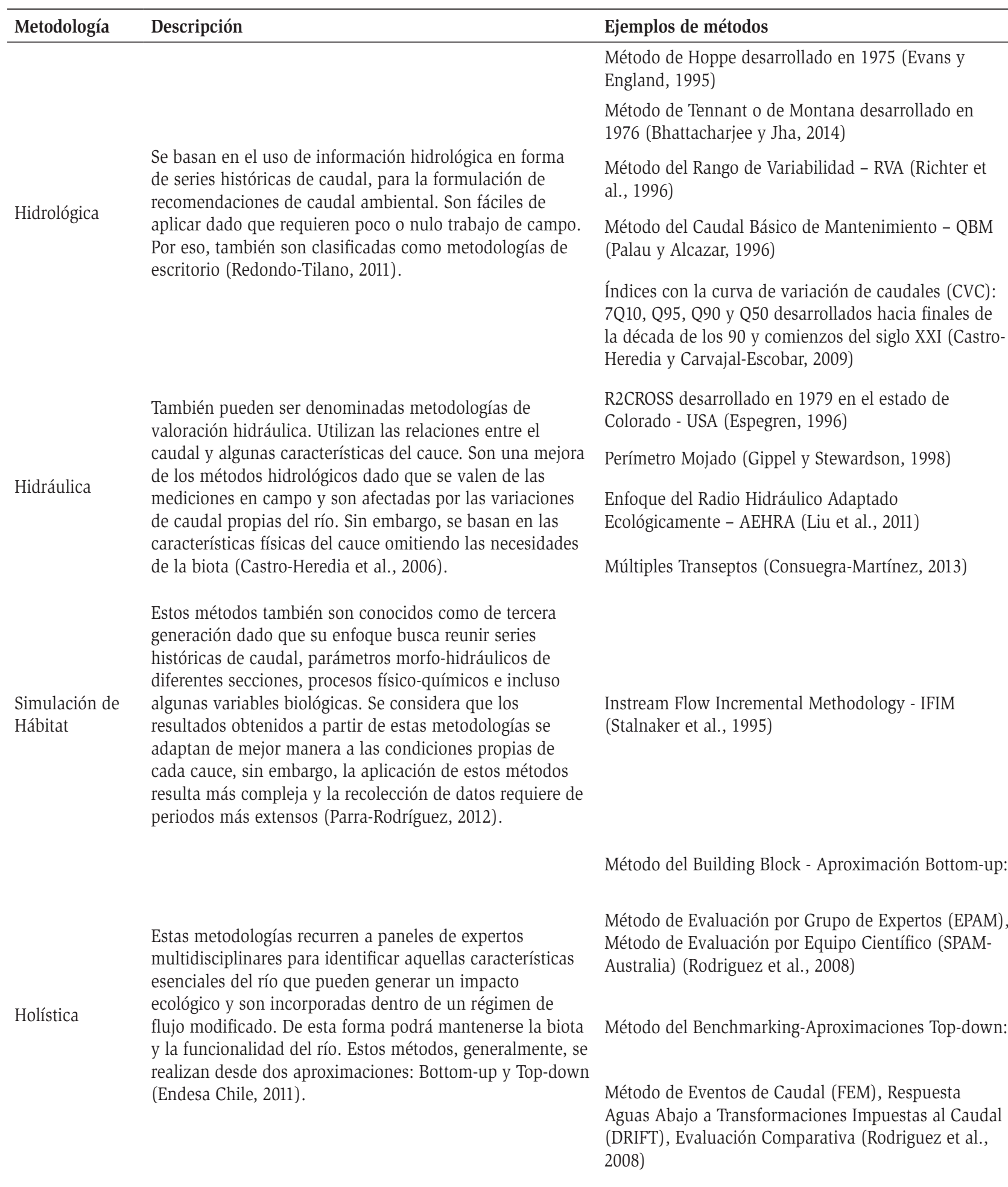

Fuente: autor. 
Finalmente, a pesar de que en Colombia aún no exista un consenso en cuanto a las metodologías utilizadas y tampoco se haya aplicado el método R2CROSS, para la determinación de caudales ecológicos, se deben destacar algunos esfuerzos, en su mayoría relacionados con la comunidad académica, de adaptar diversos métodos que son utilizados en otras latitudes para evaluar las ventajas y desventajas de su aplicación al contexto local. En la Tabla 3 se hace un recorrido cronológico por algunos de los estudios que se han adelantado en las diferentes cuencas del país, mencionando el lugar, la metodología empleada y referenciando a los autores de los estudios. En la misma se puede observar que la mayoría de casos de estudio corresponden a la implementación de metodologías de tipo hidrológico, exceptuando a Vásquez-Zapata (2009) y Parra-Rodríguez (2012) quedan por fuera de esta categoría.

\section{Método hidráulico R2CROSS}

Los métodos hidráulicos son los precursores de los métodos de tercera generación o métodos de simulación de hábitat (Palma-Raymundo, 2013) y se caracterizan por establecer una relación simple entre los índices hidráulicos del río y el hábitat de la vida acuática. El método R2CROSS, fue propuesto por primera vez por Nehring en 1979 y desde entonces, ha sido empleado exitosamente en los programas de demanda hídrica de Colorado-Estado Unidos (Ye et al., 2013). El método se basa en el supuesto de que el flujo escogido para mantener el hábitat en un rápido, asegura condiciones favorables para la biota en las demás secciones del río (Pang et al., 2012).

Cabe señalar que los rápidos son tramos de flujo poco profundo con una velocidad moderada de la corriente, que presentan algo de turbulencia superficial, una pendiente mayor y lecho de forma convexa (Linnansaari et al., 2013). También pueden definirse como tramos del río que presentan elevaciones topográficas con lecho de material grueso (Sawyer et al., 2010). Kushner (2008) manifiesta que "en términos ecológicos, los rápidos son esenciales para la producción de invertebrados acuáticos y todo el ciclo de vida de los peces residentes, proporcionando áreas para desove, incubación de huevos, alimentación y refugio.”

\section{6}

Los requerimientos de caudal para la protección del hábitat en los rápidos, están basados en flujos mínimos que garantizan el cumplimiento de los criterios establecidos para tres (03) parámetros hidráulicos:
Profundidad media, \% Perímetro mojado del cauce y Velocidad media del flujo (Parker et al., 2004). En la Tabla 4 se presentan los valores aceptables para cada uno de los parámetros hidráulicos en cuestión.

Debido a que el método está basado en recomendaciones para el mantenimiento del hábitat, debe tenerse en cuenta que estos criterios pueden ser modificados y ajustados con base en las características biológicas que se consideren pertinentes para el área de estudio, por ejemplo, condiciones del cauce, composición de las especies y calidad del hábitat acuático (Espegren, 1996).

El R2CROSS establece diferentes requerimientos de caudal con relación a las estaciones de invierno y verano. Ahora bien, Colombia es un país ubicado en la zona ecuatorial por lo cual no presenta clima estacionario así que las variaciones de temperatura se dan, principalmente, en función de la altitud, por lo tanto esta clasificación por estaciones no es aplicable a la zona de estudio. Sin embargo, a lo largo del año se presentan dos periodos con diferencias climáticas marcadas: un periodo de lluvias y un periodo seco o de estiaje (Instituto Geográfico Agustín Codazzi, 2009). En consecuencia, los resultados deberán adaptarse a los periodos mencionados anteriormente, presentando al menos un caudal para cada época del año.

Para definir el caudal ecológico durante la época de lluvias es necesario que se garantice el cumplimiento de los tres criterios establecidos por el método para los tres parámetros hidráulicos en los que se basa su aplicación (profundidad media, porcentaje de perímetro mojado del cauce y velocidad media del flujo). En el caso del periodo de estiaje, el caudal debe cumplir con al menos dos de los tres criterios presentados en la Tabla 4 (Pang et al., 2012). Esta diferenciación surgió como consecuencia de las condiciones que se viven en Colorado-USA, (lugar donde se desarrolló el método), allí los caudales de primavera / verano son mayores que los de otoño / invierno, debido a las altas tasas de deshielo y la escorrentía resultante (Kushner, 2008).

Las recomendaciones sobre el caudal ecológico dependen de datos hidráulicos y opcionalmente, biológicos. Para la recolección de los datos hidráulicos se selecciona un transecto del río, se realiza el levantamiento de la geometría del cauce y se define el caudal de descarga en esta zona. Además, pueden colectarse datos biológicos para evidenciar la presencia del ambiente natural (Espegren, 1996). 
Tabla 3. Casos de estudio sobre determinación de caudales ecológicos en Colombia.

\begin{tabular}{|c|c|c|}
\hline Río o Cuenca evaluada & Metodología aplicada & Autor (es) de la investigación \\
\hline $\begin{array}{l}\text { Cuencas media y baja de los ríos: } \\
\text { Timba, Claro, Amaime, Tuluá y } \\
\text { Pescador. }\end{array}$ & $\begin{array}{l}\text { Correlación entre comunidad biológica, parámetros físico- } \\
\text { químicos y régimen hidrológico }\end{array}$ & (Vásquez-Zapata et al., 2009) \\
\hline \multirow{8}{*}{$\begin{array}{l}47 \text { cuencas en todo el país (Río } \\
\text { Suaza, Río Bogotá, Río Saldaña, Río } \\
\text { Guarinó, Ríos Fonce y Mogoticos, Río } \\
\text { Nevado, Río Sogamoso, entre muchas } \\
\text { otras) }\end{array}$} & $\begin{array}{l}\text { Hoppe (hidrológica) } \\
\text { Tennant (hidrológica) } \\
\text { Average Base Flow - ABF (hidrológica) }\end{array}$ & \multirow{8}{*}{ (Redondo-Tilano, 2011) } \\
\hline & Range Variability Approach - RVA (hidrológica) & \\
\hline & Norma mexicana (hidrológica) & \\
\hline & Smakhtin y Anputhas (hidrológica) & \\
\hline & 7Q10 (hidrológica) & \\
\hline & UN-MAVDT (hidrológica) & \\
\hline & ENA (hidrológica) & \\
\hline & IDEAM-MAVDT (hidrológica) & \\
\hline Río Quindío & Veintidós (22) métodos hidrológicos & $\begin{array}{l}\text { (Grupo de investigación } \\
\text { CIDERA, 2011) }\end{array}$ \\
\hline $\begin{array}{l}\text { Cuenca media del río Tuluá, } \\
\text { Subcuenca del río Cauca }\end{array}$ & $\begin{array}{l}\text { Caudales Básicos de } \\
\text { Mantenimiento (CBM) }\end{array}$ & (Castro-Heredia et al., 2012) \\
\hline \multirow[t]{2}{*}{ Río Manso } & IFIM & (Parra-Rodríguez, 2012) \\
\hline & IDEAM, 2004 & \\
\hline \multirow{3}{*}{$\begin{array}{l}\text { Río Guayabero y Quebrada La } \\
\text { Reserva }\end{array}$} & Índices con la Curva de Duración de Caudales (CDC) & (Consuegra-Martínez, 2013) \\
\hline & Perímetro Mojado & \\
\hline & $\begin{array}{l}\text { IDEAM, } 2000 \\
\text { IDEAM, } 2004 \\
\text { MAVDT, } 2004\end{array}$ & \\
\hline \multirow[t]{4}{*}{ Río Chinchiná } & IDEAM, 2008 & (Ocampo et al., 2014) \\
\hline & UN-MADVT, 2008 & \\
\hline & IDEAM, 2010 & \\
\hline & MADS, 2013 & \\
\hline Río Las piedras & $\begin{array}{l}\text { Aproximación de la metodología propuesta por el MAVDT } \\
\text { en } 2008\end{array}$ & $\begin{array}{l}\text { (Casanova-O. y Figueroa-C., } \\
\text { 2015) }\end{array}$ \\
\hline
\end{tabular}

Fuente: autor. 
Tabla 4. Valores recomendados para cada variable del R2CROSS en función del ancho del río.

\begin{tabular}{cccc}
\hline $\begin{array}{c}\text { Ancho superficial del cauce } \\
\text { con caudal o descarga a } \\
\text { sección llena }(\mathrm{m})\end{array}$ & Profundidad media $(\mathrm{m})$ & $\begin{array}{c}\text { Perímetro mojado del cauce } \\
(\%)\end{array}$ & $\begin{array}{c}\text { Velocidad promedio del agua } \\
(\mathrm{m} / \mathrm{s})\end{array}$ \\
\hline $0,3-6,1$ & 0,06 & 50 & 0,3 \\
$6,2-12,2$ & $0,06-0,12$ & 50 & 0,3 \\
$12,3-18,3$ & $0,12-0,18$ & $50-60$ & 0,3 \\
$18,4-30,5$ & $0,18-0,30$ & $\geq 70$ & 0,3 \\
\hline
\end{tabular}

Fuente: Adaptado de Espegren (1996).

Hace casi una década, Kushner (2008), describió de manera detallada los procedimientos y el equipo necesario para levantar toda la información de campo requerida para la modelación de las condiciones hidráulicas en el río. Para la aplicación del método R2CROSS, es necesario calcular las pérdidas de energía por fricción en cada una de las áreas transversales seleccionadas, para esta tarea es posible utilizar el software HEC-RAS que permite simular el perfil del agua en cada rápido y de esta forma, predecir la profundidad media, la velocidad media, el porcentaje de perímetro mojado, entre otros parámetros, aguas arriba y aguas abajo del transecto evaluado en campo. Ahora bien, para conseguir simulaciones mejoradas de las condiciones hidráulicas en estas zonas de rápidos se recomienda la combinación de HEC-RAS con la medición de los parámetros hidráulicos en varias secciones transversales dentro del rápido, de manera que el modelo sea calibrado para lograr un mayor grado de precisión y fiabilidad en los resultados (Parker et al., 2004).

Después de estimar un valor de caudal ecológico, debe comprobarse la disponibilidad del mismo por medio de los registros históricos de caudal (si se tienen) o mediante la aplicación de métodos hidrológicos, así se podrá verificar la disponibilidad real de agua en el cauce. En caso de que el caudal del río sea menor al que fue establecido como caudal ecológico, debe considerarse la hipótesis de que el río se conserva con los caudales disponibles. En todo caso, deberán verificarse las condiciones específicas que pudieron originar sesgos en la aplicación del método y de ser necesario, deberá utilizarse otra metodología para la estimación del caudal ecológico (Espegren, 1996).

118

\section{Antecedentes de la aplicación del método R2CROSS}

El método R2CROSS, escogido para el desarrollo de esta investigación, no tiene tanta difusión como el método del perímetro mojado. Sin embargo, es posible encontrar algunos casos de aplicación en China y Estados Unidos. Esto puede deberse, en parte, a la simplicidad del método del perímetro mojado que lo hace más popular dentro de las metodologías hidráulicas e igualmente, al desarrollo de nuevos métodos como los de simulación de hábitats y los holísticos que son preferidos por ser más completos, pero al mismo tiempo, su aplicación resulta más compleja, costosa y demorada. La Tabla 5 presenta algunos casos en los que fue aplicado el método R2CROSS para estimar el caudal ecológico de un río.

\section{Caso de estudio: Ríos Assabet y Charles (Estados Unidos)}

Este caso desarrollado por Parker y Armstrong (2001) fue escogido no solo por tratarse del registro más antiguo presentado en la Tabla 5, sino porque resulta un buen ejemplo para ilustrar las principales cualidades del método y sus posibles fuentes de error, debido a que en esa primera evaluación que se realizó en la cuenca de los ríos Assabet y Charles, no fue posible obtener resultados concluyentes a causa de varios factores que se mencionan a continuación.

Los ríos Assabet y Charles son cuencas de drenaje adyacentes, ubicadas al Este de Massachusetts (Estados Unidos). En el río Assabet, se escogieron tres rápidos ubicados en los arroyos Elizabeth, Great y Danforth y en el río Charles, se realizaron mediciones en dos rápidos del canal principal, ubicados cerca a Mine Brook y Medway, respectivamente.

Las mediciones de caudal ecológico se realizaron con la aplicación de los métodos del perímetro mojado y el R2CROSS. Los resultados obtenidos sirvieron para establecer requerimientos preliminares para la conservación del hábitat en las secciones estudiadas. 
Tabla 5. Casos de estudio: aplicación del método R2CROSS.

\begin{tabular}{|c|c|c|c|}
\hline Río o cuenca evaluada & Métodos aplicados & Lugar & Fuente \\
\hline Ríos Assabet y Charles & $\begin{array}{l}\text { R2CROSS, Perímetro mojado } \\
\text { y Modelación del perfil } \\
\text { superficial del agua }\end{array}$ & Massachusetts (Estados Unidos) & (Parker y Armstrong, 2001) \\
\hline Río Ipswich & $\begin{array}{l}\text { Tennant, ABF Nueva } \\
\text { Inglaterra, Perímetro Mojado, } \\
\text { R2CROSS y RVA }\end{array}$ & Massachusetts (Estados Unidos) & (Armstrong et al., 2001) \\
\hline Ríos Usquepaug y Queen & $\begin{array}{l}\text { Indicadores de alteración } \\
\text { hidrológica y RVA, Tennant, } \\
\text { ABF de Nueva Inglaterra, } \\
\text { Perímetro Mojado y R2CROSS }\end{array}$ & Rhode Island (Estados Unidos) & (Armstrong y Parker, 2003) \\
\hline $\begin{array}{l}\text { Diferentes estaciones de aforo } \\
\text { de caudal }\end{array}$ & $\begin{array}{l}\text { RVA, Tennant, ABF Nueva } \\
\text { Inglaterra, Modelación del } \\
\text { perfil superficial del agua, } \\
\text { Perímetro mojado y R2CROSS }\end{array}$ & $\begin{array}{l}\text { Región de Nueva Inglaterra } \\
\text { (Estados Unidos) }\end{array}$ & (Armstrong et al., 2004) \\
\hline $\begin{array}{l}\text { Ríos Pequannok, Wanaque y el } \\
\text { Arroyo Ringwood }\end{array}$ & R2CROSS & New Jersey & (Kushner, 2008) \\
\hline Arroyos Maroon y Castle & R2CROSS & Colorado (Estados Unidos) & (Miller y Swaim, 2010) \\
\hline $\begin{array}{l}\text { Diferentes represas } \\
\text { (Baiguishan, Zhoukou, } \\
\text { Yingshang y Bangbu) }\end{array}$ & $\begin{array}{l}\text { AEHRA, Tennant, Perímetro } \\
\text { mojado y R2CROSS }\end{array}$ & Cuenca río Huai (China) & (Liu et al., 2011) \\
\hline Wei River & $\begin{array}{l}\text { WQMA, Tennant, Perímetro } \\
\text { mojado y R2CROSS }\end{array}$ & Shaanxi (China) & (Pang et al., 2012) \\
\hline Río Tarim & $\begin{array}{l}\text { Perímetro mojado, Tennant, } \\
\text { R2CROSS }\end{array}$ & Heibei (China) & (Ye et al., 2013) \\
\hline Arroyo Packers & R2CROSS y Perímetro mojado & Alaska (Estados Unidos) & (Dunker, 2015a) \\
\hline Waterfall Creek & R2CROSS y Perímetro mojado & Alaska (Estados Unidos) & (Dunker, 2015b) \\
\hline
\end{tabular}

Fuente: autor.

Esto debido a que las condiciones de flujo observadas para la calibración del modelo fueron anormales a causa de la temporada de sequía que se presentó antes de diciembre del 2001. En la Tabla 6 se presentan los resultados de caudal ecológico obtenidos para todas las estaciones ubicadas en los diferentes tramos de los ríos Assabet y Charles. Los autores del estudio también estimaron el caudal ecológico aplicando el método del perímetro mojado para comparar los resultados obtenidos con los del método R2CROSS. Las estimaciones realizadas en el arroyo Great del río Assabet, fueron descartadas debido a que la formación de remansos no permitía que esta fuera considerada una zona crítica para la aplicación de ambos métodos.

Comparados con el registro histórico de caudales disponibles en la zona (utilizando los registros históricos de caudal entre 1963-2001 tomados en la estación de aforo ubicada en el arroyo Nashoba, cerca de Acton), se puede decir que la mediana obtenida como valor de caudal para la conservación del hábitat por el método R2CROSS, es superior que el percentil 75 de las descargas diarias durante los meses de julio, agosto y septiembre (verano). Para los meses de junio y octubre, el caudal 
ecológico definido se encontraba entre el percentil 75 y la mediana de las descargas diarias. En el caso del método del perímetro mojado, los resultados obtenidos se encontraban entre el percentil 25 y el percentil 50 de los caudales diarios históricos, característicos de la época de poco caudal durante el verano (entre julio y octubre). Para el mes de junio, el valor medio de caudal ecológico se encuentra por debajo del percentil 25 del registro de caudales diarios.

Adicionalmente, se realizó un diagnóstico hidrológico del régimen natural de caudales mediante la Aproximación del Rango de Variabilidad (RVA, por sus siglas en inglés), para comparar con los resultados obtenidos mediante los métodos hidráulicos. De ese análisis se pudo observar que los rangos intercuartil de las descargas medias mensuales para las cinco estaciones, están comprendidos entre la mediana del caudal ecológico estimado con el R2CROSS y el Perímetro mojado de julio-septiembre.

Por último, se aplicó el método hidrológico de Tennant según el cual los requerimientos mínimos de caudal son del 40, 30 y 10 por ciento de la descarga promedio anual $\left(\mathrm{Q}_{\mathrm{MA}}\right)$, los cuales representan condiciones buenas, regulares y malas para el hábitat, respectivamente. Al realizar la comparación, se comprobó que la mediana del método R2CROSS se acercaba bastante a la media de Tennant $0.4 \mathrm{Q}_{\mathrm{MA}}$ mientras que la mediana del método del perímetro mojado se encuentra muy cerca a la media de Tennant $0.1 \mathrm{Q}_{\mathrm{MA}}$.

Tabla 6. Caudal ecológico de cinco (05) rápidos evaluados en los ríos Assabet y Charles en el estado de Massachusetts (Estados Unidos).

\begin{tabular}{|c|c|c|c|c|}
\hline \multirow{2}{*}{ Río } & \multirow{2}{*}{ Tramo } & \multirow{2}{*}{ Estación } & \multicolumn{2}{|c|}{ Caudal $\left(\mathrm{m}^{3} / \mathrm{s}\right)$} \\
\hline & & & R2CROSS & Perímetro mojado \\
\hline \multirow{8}{*}{ Río Assabet } & & 60,8 & 0,297 & 0,010 \\
\hline & Arroyo Danforth en Hudson & 68,7 & 0,405 & 0,002 \\
\hline & & Promedio & 0,351 & 0,006 \\
\hline & & 202,05 & 0,261 & 0,048 \\
\hline & & 213,57 & 0,340 & 0,170 \\
\hline & Arroyo Elizabeth cerca de Stow & 229,45 & 0,210 & 0,042 \\
\hline & & 241,46 & 0,425 & 0,096 \\
\hline & & Promedio & 0,309 & 0,089 \\
\hline \multirow{9}{*}{ Río Charles } & & 123,6 & 0,191 & 0,068 \\
\hline & & 164,85 & 0,248 & 0,113 \\
\hline & Arroyo Mine cerca de Franklin & 173,65 & 0,068 & 0,147 \\
\hline & & 187,25 & 0,205 & 0,028 \\
\hline & & Promedio & 0,178 & 0,089 \\
\hline & Río Charles aguas abajo de Medway & 641,1 & 1,634 & 0,246 \\
\hline & & 1481,8 & 1,286 & 0,051 \\
\hline & Río Charles aguas arriba de Medway & 1498,9 & 1,495 & 0,096 \\
\hline & & Promedio & 1,472 & 0,131 \\
\hline
\end{tabular}

Fuente: Adaptado de Parker y Armstrong (2001). 


\section{Ventajas y desventajas del método R2CROSS}

Espegren (1996) manifiestó que el método es una herramienta eficiente en cuanto al costo y al trabajo requerido, ya que se ha demostrado que los resultados obtenidos son similares a los que se producen aplicando métodos más complejos como el IFIM, con la ventaja que el trabajo de campo efectuado para el R2CROSS toma menos tiempo. Por ejemplo, según ConsuegraMartínez (2013) la implementación de la IFIM puede tardar entre 6-18 meses, mientras que el levantamiento y procesado de la información en campo requerida en el método R2CROSS se puede realizar entre 2 y 4 meses. Sin embargo, en caso de requerirse la variación interanual de los caudales ecológicos, debe tenerse en cuenta el tiempo que transcurre entre los periodos climáticos más representativos de nuestro país (época de lluvias y época de estiaje).

Otra ventaja es que, en el caso del R2CROSS, se establece una relación entre parámetros hidráulicos (perímetro mojado, profundidad de lámina de agua, velocidad, sustrato, etc.) y el bienestar ecosistémico, facilitando la evaluación de caudales ecológicos con modelaciones realizadas a partir de la información base levantada en campo. Entonces, aunque el método fue diseñado en Colorado (Estados Unidos), una zona donde existen las estaciones climáticas, puede ser adaptado a Colombia siempre y cuando se tenga en cuenta que existe una diferencia significativa entre la oferta de agua de los ríos durante los periodos de lluvia y de sequía. Por consiguiente, es importante definir un plan de trabajo en campo que considere las condiciones durante la época de estiaje y la época lluviosa.

Otro aspecto importante es que el método implica una inversión intermedia dado que sus costos son mayores que las metodologías hidrológicas pero menores que las holísticas y de simulación ecohidráulica (Consuegra-Martínez, 2013). Adicionalmente, puede ser lo suficientemente flexible para adaptarse a los requerimientos de las diferentes especies de un ecosistema (excluyendo el componente ribereño: vegetación ripiaria y fauna) y a las actividades o estadio de desarrollo de las mismas (Alcazar-Montero, 2007).
Tal como lo destaca Parker et al. (2004) una de las principales ventajas de los métodos hidráulicos es que se basan en observaciones de campo y no dependen de un registro histórico de caudales y/o precipitaciones. Esto puede ser particularmente útil en zonas donde se presentan condiciones hidrológicas especiales y donde no existen estaciones meteorológicas, pluviométricas e hidrológicas que permitan tener un registro histórico de caudales para describir las características de la cuenca. En Colombia, por ejemplo, la información hidrológica de la red de estaciones del IDEAM no siempre resulta adecuada debido que no cumple con la cantidad, calidad, consistencia y agregación temporal necesarias para una adecuada descripción de las condiciones de la cuenca. Adicionalmente, en muchas cuencas no existen estaciones meteorológicas cercanas (ÁlvarezMontoya, 2013).

Lo anterior, a pesar de ser una ventaja, debe ser tomado con precaución debido que los registros históricos de caudal son un mecanismo de ajuste y control para los caudales ambientales estimados a partir de los métodos hidráulicos, puesto que permiten comprobar que éstos últimos se encuentren dentro del régimen natural de caudales de la cuenca. Es decir, que el caudal ecológico estimado esté disponible de forma natural en el río evaluado.

La correcta selección del tramo de estudio también es un factor determinante en la obtención de resultados confiables, específicamente, los tramos seleccionados deben tratarse de rápidos donde la sección sea ancha y poco profunda, con lecho conformado por material con una granulometría entre gravas y cantos rodados (Parker et al., 2004; Ye et al., 2013). Por otra parte, la presencia de grandes rocas expuestas que dividen el canal de banca llena, restos de madera, socavaciones en la banca y orillas o lechos alterados, entre otros aspectos, conllevan a realizar estimaciones alejadas las necesidades reales del río (Parker et al., 2004; Dunker, 2015a).

Una de las limitaciones de los métodos hidráulicos $\mathrm{y}$, en consecuencia, del método R2CROSS, es que la fiabilidad de los resultados depende de que su aplicación se realice en cauces naturales y no en ríos regulados 
o ampliamente intervenidos (i.e. ensanchamiento y estrechamiento de tramos, refuerzo de lechos y orillas con escolleras) y contaminados, en cuyo caso sería más pertinente una metodología que considere la calidad del agua como factor decisivo para la estimación del caudal ambiental (Pang et al., 2012).

No obstante, algunos autores mencionan que los métodos hidráulicos resultan muy simplistas al considerar que una o varios parámetros hidráulicos son suficientes para determinar los requerimientos de caudal de las distintas especies, ya que con esto se están dejando a un lado "las relaciones complejas de un sistema dinámico y dependiente de otras variables y especies” (Álvarez-Montoya, 2013). Son varios los trabajos que coinciden en que lo anterior es una de las principales desventajas de la aplicación de este tipo de métodos de valoración hidráulica (Alcazar-Montero, 2007; Castro-Heredia y Carvajal-Escobar, 2009; CarvajalEscobar, 2010; Consuegra-Martínez, 2013; Linnansaari et al., 2013). En cualquier caso, la información levantada para el R2CROSS, sirve como insumo para la implementación de métodos más complejos como por ejemplo el IFIM, la Metodología para la Estimación y Evaluación del Caudal Ambiental en Proyectos que requieren Licencia Ambiental y otros métodos holísticos.

En la Tabla 7 se resumen las principales ventajas y desventajas para la aplicación de los métodos hidráulicos y del método R2CROSS. Algunas de ellas son válidas para cualquier método hidráulico, mientras que otras son específicas para el método R2CROSS y se identificaron con base a las condiciones específicas de Colombia, razón por la cual no pueden generalizarse a otras regiones del mundo.

\section{Consideraciones finales}

Si bien es cierto que Colombia cuenta con una metodología propia para estimar el caudal ecológico, la difusión de la misma ha sido bastante limitada bien sea por falta de registros bibliográficos o porque las restricciones técnicas en cuanto a la disponibilidad de datos hidrológicos han resultado un impedimento para quienes hayan considerado aplicar el método. Así, por ejemplo, al momento de elaborar este artículo, se 122

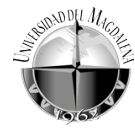

populares (ver Tabla 3). Incluso después del 2013, algunas Corporaciones Autónomas Regionales (CAR) como CORNARE seguían permitiendo, dentro de los términos de referencia para la elaboración de estudios de impacto ambiental para centrales hidroeléctricas, una libre selección del método para estimar caudales ambientales (CORNARE y Grupo GELAG, 2013).

Por otro lado, es importante tener en cuenta que la metodología del ANLA está enfocada en proyectos que requieren licencia ambiental y que involucran el aprovechamiento del recurso hídrico, por ejemplo, centrales hidroeléctricas, embalses, trasvases de cuenca, entre otros. En la práctica, el desarrollo de este tipo de proyectos implica que existe la disponibilidad de recursos técnicos y económicos por parte de las firmas interesadas en el licenciamiento del proyecto; contrario a lo que ocurre con las entidades públicas encargadas de la administración del recurso hídrico, como es el caso de las CAR, quienes tienen a cargo dicha función pero, al mismo tiempo, cuentan con limitantes técnicas (falta de equipos e instrumentación, ausencia de profesionales especialistas en manejo del recurso, entre otros) y, principalmente, económicas y temporales.

Por lo anterior, para las corporaciones y/o autoridades ambientales, encargadas de la formulación de los POMCAs, se vuelve imperativo contar con un método que sea al mismo tiempo práctico y eficiente, sobre todo si se tiene en cuenta que dentro de la jurisdicción de las CAR puede haber numerosos ríos que no cuentan con una línea base y cuya información hidrológica es inexistente o con una resolución temporal muy pobre para estimar los caudales ecológicos con "métodos de escritorio". En consecuencia, el método R2CROSS podría ser utilizado como una herramienta alternativa para estimar el caudal ecológico en Colombia teniendo en cuenta, además, que los resultados del mismo han resultado lo suficiente conservadores para garantizar la preservación de los ecosistemas y que, además, no depende de la disponibilidad de datos en estaciones hidrológicas.

Inicialmente, el método sería aplicable a ríos susceptibles de ser intervenidos en el corto plazo con actividades como concesiones de agua, vertimientos, transvases de cuenca, construcción de centrales hidroeléctricas, represas y cualquier otra que implique el aprovechamiento del recurso hídrico. A mediano y largo plazo, la información recopilada con este método serviría como insumo para estimar caudales con otros métodos más completos como el de la ANLA que, 
ciertamente, resultan más efectivos a la hora de estimar el caudal ambiental de un río, pero que resultan de difícil aplicación en las condiciones que se han mencionado a lo largo de este artículo. En resumen, el R2CROSS es un puente que facilitaría la transición entre el uso de los métodos hidrológicos a los holísticos.

Tabla 7. Ventajas y desventajas de la aplicación del método R2CROSS.

\begin{tabular}{ll}
\hline Ventajas & Desventajas \\
\hline
\end{tabular}

Establece una relación entre parámetros hidráulicos y el bienestar ecosistémico.

Se asumen vínculos ecológicos que no se comprueban.

Tiempos de trabajo relativamente cortos (2 a 4 meses).

Son específicos al lugar de trabajo, a los transeptos o secciones hidráulicas trabajadas, evitando sesgos por extrapolar información de estaciones hidrológicas.

Puede ser aplicado en cuencas perturbadas hidrológicamente y en sitios donde no hay aforo de caudal, registros de precipitación, limnígrafos o limnímetros.

Buena relación Costo-Beneficio al obtener resultados similares al método IFIM, pero con costos y tiempos de aplicación menores.

Son métodos recomendados cuando la información disponible de los sistemas rivereños es insuficiente.

La información levantada para el método sirve como insumo para la implementación de métodos más complejos.
Para establecer un régimen de caudales ecológicos, es necesario realizar diferentes campañas de campo.

Implica una inversión intermedia, dado que sus costos son mayores que las metodologías hidrológicas pero menores que las holísticas y de simulación ecohidráulica.

La selección del periodo para realizar el trabajo de campo y la localización de secciones transversales representativas de toda la corriente, son trabajos críticos para garantizar la confiabilidad de los resultados que se obtengan.

La variación de las características en diferentes rápidos de la zona del río a estudiar, puede llegar a tener una influencia significativa como fuente de error.

No es recomendable su aplicación en ríos anastomosados (trenzados), intervenidos y muy contaminados.

Es recomendable comparar los requerimientos de caudal determinados con respecto a la información hidrológica.

Fuente: Autor con información de Rojas-Vásquez y Tarambis-Enríquez (2012); Consuegra-Martínez (2013); Linnansaari et al. (2013).

Es importante aclarar que el R2CROSS más que un método para estimar caudales ambientales es un método para determinar el caudal ecológico del río (ver diferencias en Tabla 1), toda vez que centra su atención en el medio biofísico, dejando a un lado los aspectos socioeconómicos. El método es la base de un proceso de caracterización de las cuencas que tiene como propósito preservar el recurso para, posteriormente, buscar una integración con el uso que le dan las comunidades al mismo (recreación, riego, abastecimiento, fuente de alimentos, etc.). Dicho de otra manera, lo que se busca al aplicar este método es conocer en el corto plazo, el caudal que garantiza el flujo de agua y la preservación del ecosistema (a partir de los valores del método), para definir las restricciones de uso de acuerdo al caudal disponible (igual al caudal total menos el caudal 
ecológico) y, posteriormente, a mediano plazo, dentro del POMCA puedan diseñarse medidas que permitan un aprovechamiento sostenible del recurso.

Finalmente, para la implementación del método en Colombia, se sugiere realizar una prueba piloto en cuencas con suficiente información hidrológica de tal forma que los resultados obtenidos puedan ser comparados con caudales estimados mediante métodos hidrológicos y a su vez, pueda verificarse la disponibilidad del recurso de acuerdo al régimen natural de caudales del río. Solo de esta forma se podría validar o descartar definitivamente la aplicación del R2CROSS en condiciones locales.

\section{BIBLIOGRAFÍA}

Agualimpia-Dualiby, Y. del C. y Castro-Méndez, C.E. 2006. Metodologías para la determinación de los caudales ecológicos en el manejo de los recursos hídricos. Tecnogestión 3(1): 3-13.

Alcazar-Montero, J. 2007. El método del Caudal Básico para la determinación de caudales de mantenimiento. Aplicación a la Cuenca del Ebro. Universidad de Lleida, España.

Álvarez-Montoya, G.H. 2013. Análisis de los criterios físicos de la metodología para la estimación de caudales ambientales del Ministerio de Ambiente y Desarrollo Sostenible. Casos: proyectos hidroeléctricos Mayaba y Santo Domingo. Tesis de Pregrado, Escuela de Ingeniería de Antioquia, Envigado, Colombia.

Armstrong, D.S. y Parker, G.W. 2003. Assessment of Habitat and Streamflow Requirements For Habitat Protection, Usquepaug - Queen River, Rhode Island, 1999 - 2000. Northborough, Massachusetts, United States.

Armstrong, D.S., Parker, G.W. y Richards, T.A. 2004. Evaluation of Streamflow Requirements for Habitat Protection by Comparison to Streamflow Characteristics at Index Streamflow-Gaging Stations in Southern New England. Reston Virginia, United States.

Armstrong, D.S., Richards, T.A. y Parker, G.W. 2001. Assessment of Habitat, Fish Communities and Streamflow Requirements for Habitat Protection, Ipswich River,

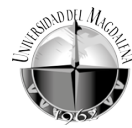

Resources Investigations Report 01-4161._URL:_http:// pubs.usgs.gov/wri/wri01-4161/. Consultado: 13 de febrero de 2015.
Autoridad Nacional de Licencias Ambientales. 2013. Metodología para la estimación y evaluación del caudal ambiental en proyectos que requieren licencia ambiental. Ministerio de Ambiente y Desarrollo Sostenible, Bogota D.C.

Bhattacharjee, A. y Jha, R. 2014. Environmental flows State-of-the-art with details. Assessment of a typical river basin of India, Chennai, India. International Conference on Innovative Technologies and Management for Water Security._URL:_https://www.researchgate.net/ profile/Anuran_Bhattacharjee/publication/260245950_ ENVIRONMENTAL_FLOWS_STATE-OF-THE-ART_WITH_ DETAILS_ASSESSMENT_OF_A_TYPICAL_RIVER_BASIN_ OF_INDIA/links/00b7d5304bb1d88d4e000000.pdf/ download? version $=$ va. Consultado: 04 de marzo de 2016.

Carvajal-Escobar, Y. 2010. Dimensiones para usar metodologías en la estimación de caudales ambientales en Colombia. Respuestas 15(2): 34-46.

Casanova-O., J.F. y Figueroa-C., A. 2015. Determinación del caudal ambiental y su relación con variables indicadoras de calidad del recurso hídrico. Luna Azul 40: 5-24 http://200.21.104.25/lunazul/downloads/Lunazul40_2. pdf. Consultado: 14 de marzo de 2015.

Castro-Heredia, L.M. y Carvajal-Escobar, Y. 2009. Metodologías para determinar el caudal ambiental. En: Cantera-Kintz, J.R., Carvajal-Escobar, Y. y Castro-Heredia, L.M., Editores. Caudal ambiental. Conceptos, experiencias y desafíos. Programa editorial Universidad del Valle, Cali.

Castro-Heredia, L.M., Carvajal-Escobar, Y. y CéspedesLópez, G. 2012. Aplicación de la metodología de caudales básicos de mantenimiento en la Cuenca Media del río Tuluá - Subcuenca del Río Cauca. Revista Tecnura 16(31): 33-44.

Castro-Heredia, L.M., Carvajal-Escobar, Y. y MonsalveDurango, E.A. 2006. Enfoques teóricos para definir el caudal ambiental. Revista Ingeniería e Investigación 10(2): 179-195.

Consuegra-Martínez, C.S.M. 2013. Síntesis metodológica para la obtención de caudales ecológicos (Qe), resultados y posibles consecuencias. Trabajo de grado para optar al título de Especialista en recursos hidráulicos y medio ambiente, Escuela Colombiana de Ingeniería Julio Garavito, Bogotá D.C, Colombia http://repositorio.escuelaing.edu. co/bitstream/001/75/1/DOCUMENTO \% 20-\%20FINAL. pdf. Consultado: 20 de febrero de 2015. 
CORNARE y Grupo GELAG. 2013. Términos de referencia. Sector Energía. Estudio de impacto ambiental. Construcción y operación de centrales hidroeléctricas generadoras, Colombia._URL:_http://www.cornare.gov.co/TramitesAmbientales/TR/TR-EIA-CENTRALES.pdf. Consultado: 11 de octubre de 2015.

Díez-Hernández, J.M. 2005. Bases metodológicas para el establecimiento de caudales ecológicos en el ordenamiento de cuencas hidrográficas. Ingeniería y Competitividad 7(2): 11-18.

Dunker, B. 2015a. Packers Creek Hydroelectric Project, Hydrology and Fish Surveys, 2013. Technical report No. 14-09. Alaska Department of Fish and Game, Division of Habitat, United States.

Dunker, B. 2015b. Waterfall Creek Hydroelectric Project, Hydrology and Fish Surveys, 2013. Technical report No. 14-10. Alaska Department of Fish and Game, Division of Habitat, United States.

Endesa Chile. 2011. Introducción al cálculo de caudales ecológicos. Un análisis de las tendencias actuales. Empresa Nacional de Electricidad S.A., Santiago de Chile.

Espegren, G.D. 1996. Development of Instream flow recommendations in Colorado using R2CROSS. Water Conservation Board, Department of Natural Resources, Water Rights Investigations Section, Denver, Colorado, United States.

Evans, J.W. y England, R.H. 1995. A Recommended Method to Protect Instream Flows in Georgia. Georgia Department of Natural Resources, Georgia, United States.

Gippel, C.J. y Stewardson, M.J. 1998. Use of wetted Perimeter in Defining Minimum Environmental Flows. Regulated Rivers: Research y Management 14(November): 53-67.

Grupo de investigación CIDERA. 2011. Estimación de caudales ecológicos mediante métodos hidrológicos e hidráulicos en la UMC Río Quindío, Armenia, Colombia.

IDEAM. 2015. Estudio Nacional del Agua 2014. IDEAM, Bogotá, D.C., Colombia.

Instituto Geográfico Agustín Codazzi. 2009. Descripción del medio biofísico. En: Estudio general de suelos y zonificación de tierras. Departamento del Magdalena. Escala 1:100.000. 55-142.

Izquierdo-Santacruz, M.L. y Madroñero-Palacios, S.M. 2014. Régimen de caudal ecológico, herramienta de gestión para conservar la biota acuática. Ciencia e ingeniería neogranadina 23(2): 77-94.

Kushner, R. 2008. 2008 Stream Flow Analysis Using R2CROSS. Newfoundland, New Jersey, United States.

Linnansaari, T., Monk, W.A., Baird, D.J. y Curry, R.A. 2013. Review of approaches and methods to assess Environmental Flows across Canada and internationally, Canada._URL:_http://www.dfo-mpo.gc.ca/csas-sccs/ Publications/ResDocs-DocRech/2012/2012_039-eng.html. Consultado: 05 de abril de 2015.

Liu, C., Zhao, C., Xia, J., Sun, C., Wang, R. y Liu, T. 2011. An instream ecological flow method for datascarce regulated rivers. Journal of Hydrology 398(1-2): 17-25 http://dx.doi.org/10.1016/j.jhydrol.2010.11.026. Consultado: 11 de octubre de 2015

Liu, J., Liu, Q. y Yang, H. 2016. Assessing water scarcity by simultaneously considering environmental flow requirements, water quantity, and water quality. Ecological Indicators 60: 434-441 http://www.sciencedirect.com/ science/article/pii/S1470160X15004045. Consultado: 14 de marzo de 2015.

Madroñero-Palacios, S.M. y Mafla-Chamorro, F.R. 2013. Caudales ecológicos y su relación con el cambio y la variabilidad climática. Revista Unimar (61): 61-77.

Magdaleno-Mas, F. 2004. Caudales ecológicos: conceptos básicos, métodos de cálculo y nuevas interpretaciones. Ingeniería Civil 135: 81-92.

Miller, W.J. y Swaim, K.M. 2010. Castle Creek Hydroelectric Plant Environmental Report. Fort Collins, Colorado, United States.

Ministerio de Ambiente Vivienda y Desarrollo Territorial. 2010. Política Nacional para la Gestión Integral del Recurso Hídrico. Ministerio de Ambiente Vivienda y Desarrollo Territorial, Bogotá D.C., Colombia.

O’Keeffe, J. y Quesne, T.Le. 2010. Cómo conservar los ríos vivos. Guía sobre los caudales ecológicos., Gland (Suiza): World Wildlife Fund._URL:_http://www.agua. unam.mx/humedales/assets/materialdifusion/WWF_ ComoConservarLosRiosVivos.pdf. Consultado: 13 de febrero de 2015.

Ocampo, O., Vélez, J.J., Giraldo, D.C. y Sánchez, E. 2014. Caudales ecológicos y ambientales en cuencas andinas colombianas. XXV Congreso Latinoamericano de Hidráulica. 
Palau, A. y Alcazar, J. 1996. The basic flow: An alternative approach to calculate minimum environmental instream flows. Ecohydroulique 2000: 547-558.

Palma-Raymundo, M.L. 2013. Determinación del caudal ecológico: impacto económico en el usuario agrícola de la cuenca del Río Yautepec, Estado de Morelos. Tesis de Maestría, Institución de enseñanza e investigación en ciencias agrícolas, Puebla, Puebla, México http://www. biblio.colpos.mx:8080/xmlui/bitstream/10521/2208/1/ Palma_Raymundo_ML_MC_EDAR_2013.pdf. Consultado: 04 de marzo de 2015.

Pang, B., Xu, Z.X. y Wu, W. 2012. Estimation of the ecological base flow of Wei River in Shaanxi province. Procedia Environmental Sciences 13: 1559-1568.

Parker, G.W. y Armstrong, D.S. 2001. Preliminary Assessment of Streamflow Requirements for Habitat Protection for Selected Sites on the Assabet and Charles Rivers, Eastern Massachusetts. Northborough, Massachusetts, United States.

Parker, G.W., Armstrong, D.S. y Richards, T.A. 2004. Comparison of methods for determining streamflow requirements for aquatic habitat protection at selected sites on the Assabet and Charles Rivers, eastern Massachusetts, 2000-02. Reston (Virginia). United States._URL:_http:// pubs.usgs.gov/sir/2004/5092/pdf/sir_2004_5092_old.pdf. Consultado: 04 de marzo de 2015.

Parra-Rodríguez, E.A. 2012. Modelamiento y manejo de las interacciones entre la hidrología, la ecología y la economía en una cuenca hidrográfica para la estimación de caudales ambientales. Tesis de maestría, Universidad Nacional de Colombia, Medellín, Colombia.

Redondo-Tilano, S.A. 2011. Incertidumbre hidrológica en la estimación de caudales ambientales mediante metodologías basadas en series históricas. Tesis de Maestría, Universidad Nacional de Colombia, Bogotá, Colombia.

Richter, B.D., Baumgartner, J.V. y Powell, J. 1996. A method for assesing hydrologic alteration within ecosystems. Conservation Biology 10(4): 1163-1174.
Rodríguez, E.A., Pinilla, G., Camacho, L.A., Medina, M.P., Ramírez, A. y Bernal, E. 2008. Metodología para la estimación del caudal ambiental en proyectos licenciados. Bogotá D.C., Colombia.

Rodríguez-Gallego, L., Chreties, C., Crisci, M., Fernández, M., Colombo, N., Lanzilotta, B., Saravia, M., Neme, C., Sabaj, V. y Conde, D. 2011. Fortalecimiento del concepto de Caudales Ambientales como Herramienta para la Gestión Integrada de los Recursos Hídricos. Informe Final, Acuerdo PNUMA y Vida Silvestre Uruguay, Uruguay.

Rojas-Vásquez, D.R. y Tarambis-Enriquez, H.D. 2012. Bases de diseño de estructuras en diques transversales para la captación y tránsito del caudal ecológico. Tesis de pregrado, Escuela Politécnica Nacional, Quito, Ecuador.

Sawyer, A.M., Pasternack, G.B., Moir, H.J. y Fulton, A.A. 2010. Riffle-pool maintenance and flow convergence routing observed on a large gravel-bed river. Geomorphology 114(3): 143-160.

Stalnaker, C., Lamb, B.L., Henriksen, J., Bovee, K. y Bartholow, J. 1995. The Instream Flow Incremential Methodology: A primer for IFIM. U.S. Department of the interior. National biological service._URL: https://www.fort.usgs.gov/sites/default/files/products/ publications/2422/2422.pdf. Consultado: 04 de marzo de 2016.

Vásquez-Zapata, G.L., Ramírez-Lamus, J.C., BlancoLibreros, J.F., Navarrete-Gálvez, A.M., Cantera-Kintz, J.C. y Montoya-Montoya, P.N. 2009. Casos de estudio en la determinación del régimen de caudal ambiental. Caso 4: Determinación de los caudales ecológicos para el normal desarrollo de la biota acuática, en las cuencas media y baja de los ríos: Timba, Claro, Amaime, Tulua y Pescador, en el departamento del Valle del Cauca. En: Cantera-Kintz, J.R., Carvajal-Escobar, Y. y Castro-Heredia, L.M., Editores. Caudal ambiental. Conceptos, experiencias y desafíos. Programa editorial Universidad del Valle, Cali.

Ye, Z., Shen, Y. y Chen, Y. 2013. Multiple methods for calculating minimum ecological flux of the desiccated Lower Tarim River, Western China. Ecohydrology 6(6): 1040-1047. 